\title{
Independence Numbers in Trees
}

\author{
Min-Jen Jou ${ }^{1}$, Jenq-Jong Lin ${ }^{2}$ \\ ${ }^{1}$ Department of Information Technology, Ling Tung University, Taichung Taiwan \\ ${ }^{2}$ Department of Finance, Ling Tung University, Taichung Taiwan \\ Email: mjjou@teamail.tu.edu.tw, jilin@teamail.tu.edu.tw
}

Received 3 March 2015; accepted 14 July 2015; published 17 July 2015

Copyright (C) 2015 by authors and Scientific Research Publishing Inc.

This work is licensed under the Creative Commons Attribution International License (CC BY).

http://creativecommons.org/licenses/by/4.0/

(c) (i) Open Access

\section{Abstract}

The independence number $\alpha(G)$ of a graph $G$ is the maximum cardinality among all independent sets of $G$. For any tree $T$ of order $n \geq 2$, it is easy to see that $\left\lceil\frac{n}{2}\right\rceil \leq \alpha(T) \leq n-1$. In addition, if there are duplicated leaves in a tree, then these duplicated leaves are all lying in every maximum independent set. In this paper, we will show that if $T$ is a tree of order $n \geq 4$ without duplicated leaves, then $\alpha(T) \leq\left\lfloor\frac{2 n-1}{3}\right\rfloor$. Moreover, we constructively characterize the extremal trees $T$ of order $n \geq 4$, which are without duplicated leaves, achieving these upper bounds.

\section{Keywords}

Independent Set, Independence Number, Tree

\section{Introduction}

All graphs considered in this paper are finite, loopless, and without multiple edges. For a graph $G$, we refer to $V(G)$ and $E(G)$ as the vertex set and the edge set, respectively. The cardinality of $V(G)$ is called the order of $G$, denoted by $|G|$. The (open) neighborhood $N_{G}(x)$ of a vertex $x$ is the set of vertices adjacent to $x$ in $G$, and the close neighborhood $N_{G}[x]$ is $N_{G}(x) \cup\{x\}$. A vertex $x$ is said to be a leaf if $\left|N_{G}(x)\right|=1$. A vertex $v$ of $G$ is a support vertex if it is adjacent to a leaf in $G$. Two distinct vertices $u$ and $v$ are called duplicated if $N_{G}(u)=N_{G}(v)$. Note that $u$ and $v$ are duplicated vertices in a tree, and then they are both leaves. The $n$-path $P_{n}$ is the path of order $n \geq 1$. For a subset $A \subseteq V(G)$, the induced subgraph induced by $A$ is the graph $\langle A\rangle_{G}$ with vertex set $A$ and the edge set $E\left(\langle A\rangle_{G}\right)=\{u v \in E(G): u \in A$ and $v \in A\}$, the deletion of $A$ from $G$ is the graph $G-A$ by removing all vertices in $A$ and all edges incident to these vertices and the complement of $A$ is the set $A^{c}=V(G) \backslash A$. For notation and terminology in graphs we follow [1] in general. 
A set $I \subseteq V(G)$ is an independent set of $G$ if no two vertices of $I$ are adjacent in $G$. The independence number $\alpha(G)$ of $G$ is the maximum cardinality among all independent sets of $G$. If $I$ is an independent set of $G$ with cardinality $\alpha(G)$, we call $I$ an $\alpha$-set of $G$. If $I$ is an $\alpha$-set of $G$ containing all leaves of $G$, we call $I$ an $\alpha_{L}$-set of $G$.

The independence problem is to find an $\alpha$-set in $G$. The problem is known to be NP-hard in many special classes of graphs. Over the past few years, several studies have been made on independence (see [2]-[6]). For any tree $T$ of order $n \geq 2$, it is easy to see that $\left\lceil\frac{n}{2}\right\rceil \leq \alpha(T) \leq n-1$. In addition, if there are duplicated leaves in a tree, then these duplicated leaves are all lying in every maximum independent set. In this paper, we will show that if $T$ is a tree of order $n \geq 4$ without duplicated leaves, then $\alpha(T) \leq\left\lfloor\frac{2 n-1}{3}\right\rfloor$. Moreover, we constructively characterize the extremal trees $T$ of order $n \geq 4$, which are without duplicated leaves, achieving these upper bounds.

\section{The Upper Bound}

In this section, we will show a sharp upper bound on the independence number of a tree $T$ without duplicated leaves.

Lemma 1 If $H$ is an induced subgraph of $G$, then $\alpha(H) \leq \alpha(G)$.

Proof. If $S$ is an $\alpha$-set of $H$, then $S$ is an independent set of $G$. It follows that $\alpha(H)=|S| \leq \alpha(G)$.

Lemma 2 ([4]) If $T$ is a tree of order $n \geq 1$, then $\alpha(T) \geq\left\lceil\frac{n}{2}\right\rceil$.

Lemma 3 ([5]) If $T$ is a tree of order $n \geq 3$, then there exists an $\alpha_{L}$-set of $T$.

Lemma 4 For an integer $n \geq 4, \alpha\left(P_{n}\right)=\left\lceil\frac{n}{2}\right\rceil \leq\left\lfloor\frac{2 n-1}{3}\right\rfloor$.

Proof. It is straightforward to check that $\left\lceil\frac{n}{2}\right\rceil \leq\left\lfloor\frac{2 n-1}{3}\right\rfloor$ for $n \geq 4$. Let $P_{n}: v_{1}-v_{2}-\cdots-v_{n}$. Since $P_{n}$ is a tree of order $n \geq 4$, by Lemma 2, we have that $\alpha\left(P_{n}\right) \geq\left\lceil\frac{n}{2}\right\rceil$. Suppose that there exists an independent set $I$ of $P_{n}$ with $|I| \geq\left\lceil\frac{n}{2}\right\rceil+1$, then there exists $i, 1 \leq i \leq n-1$, such that $v_{i} \in I$ and $v_{i+1} \in I$. This is a contradiction, therefore we obtain that $\alpha\left(P_{n}\right)=\left\lceil\frac{n}{2}\right\rceil$.

Theorem 1 If $T$ is a tree of order $n \geq 4$ without duplicated leaves, then $\alpha(T) \leq\left\lfloor\frac{2 n-1}{3}\right\rfloor$.

Proof. We prove it by induction on $n \geq 4$. By Lemma 4 and $T$ is a tree without duplicated leaves, it's true for all $n \leq 6$. For all $n \geq 7$ we assume that the assertion is true for all $n^{\prime}<n$. Suppose that $T$ is a tree of order $n \geq 7$ without duplicated leaves and $x$ is a leaf lying on a longest path of $T$. Let $y \in N_{T}(x)$. Since $T$ has no duplicated leaves, this implies that $d_{T}(y)=2$, say $N_{T}(y)=\{x, z\}$. Let $T^{\prime}=T-N_{T}[x]$, then $T^{\prime}$ is a tree of order $n-2$. For the case in which $T^{\prime}$ has no duplicated leaves, by induction hypothesis, we have that $\alpha\left(T^{\prime}\right) \leq\left\lfloor\frac{2(n-2)-1}{3}\right\rfloor=\left\lfloor\frac{2 n-5}{3}\right\rfloor$. Since an $\alpha$-set of $T^{\prime}$, together with $\{x\}$, form an $\alpha$-set of $T$. Therefore we obtain that $\alpha(T)=\alpha\left(T^{\prime}\right)+1 \leq\left\lfloor\frac{2 n-5}{3}\right\rfloor+1=\left\lfloor\frac{2 n-2}{3}\right\rfloor \leq\left\lfloor\frac{2 n-1}{3}\right\rfloor$. For the other case in which $T^{\prime}$ has duplicated leaves $z$ and $z^{\prime}$, then $T^{\prime \prime}=T^{\prime}-\{z\}$ is a tree of order $n-3 \geq 4$ without duplicated leaves. By induction hypothesis, we have that $\alpha\left(T^{\prime \prime}\right) \leq\left\lfloor\frac{2(n-3)-1}{3}\right\rfloor=\left\lfloor\frac{2 n-7}{3}\right\rfloor$. Since an $\alpha_{L}$-set of $T^{\prime \prime}$, together with $\{x, z\}$, form 
an $\alpha$-set of $T$. Therefore, we obtain that $\alpha(T)=\alpha\left(T^{\prime \prime}\right)+2 \leq\left\lfloor\frac{2 n-7}{3}\right\rfloor+2=\left\lfloor\frac{2 n-1}{3}\right\rfloor$. Hence we conclude that $\alpha(T) \leq\left\lfloor\frac{2 n-1}{3}\right\rfloor$.

Note that the result in Theorem 1 is sharp and some such $T$ are illustrated below.

\section{Extremal Trees}

Let $\mathscr{T}(n)$ be the class of all trees $T$ of order $n \geq 4$ without duplicated leaves such that $\alpha(T)=\left\lfloor\frac{2 n-1}{3}\right\rfloor$. We will constructively characterize these extremal trees. Let $L(T)$ and $U(T)$, respectively, denote the collections of all leaves and all support vertices of $T$. First, we define four operations on a tree $T$ of order $n \geq 4$ as follows, where $I$ is an $\alpha_{L}$-set of $T$.

Operation O1. Join a vertex $u \in I$ of $T$ to a vertex $v_{1}$ of $P_{1}$ such that $I_{O 1}=(I-\{u\}) \cup\left\{v_{1}\right\}$, where $|T|=n \equiv 2 \quad(\bmod 3)$.

Operation O2. Join a vertex $u \in I^{c} \backslash U(T)$ of $T$ to a vertex $v_{1}$ of $P_{1}$ such that $I_{O 2}=I \cup\left\{v_{1}\right\}$, where $|T|=n \equiv 0,1 \quad(\bmod 3)$.

Operation O3. Join a vertex $u$ of $T$ to a leaf $v_{2}$ of $P_{2}$ (say $P_{2}: v_{1}-v_{2}$ ) such that $I_{O 3}=I \cup\left\{v_{1}\right\}$, where $|T|=n \equiv 1,2 \quad(\bmod 3)$.

Operation O4. Join a vertex $u \in I^{c}$ of $T$ to a leaf $v_{3}$ of $P_{3}$ (say $P_{3}: v_{1}-v_{2}-v_{3}$ ) such that $I_{O 4}=I \cup\left\{v_{1}, v_{3}\right\}$.

Lemma 5 Suppose that $T \in \mathscr{T}(n)$ for $n \geq 4$. If I is an $\alpha_{L}$-set of $T$, then

$$
\left|I^{c} \backslash U(T)\right| \leq \begin{cases}0, & \text { if } n \equiv 2(\bmod 3), \\ 1, & \text { if } n \equiv 1(\bmod 3), \\ 2, & \text { if } n \equiv 0(\bmod 3) .\end{cases}
$$

Proof. It's true for all $n \leq 6$. So we assume that $n \geq 7$. Since $I$ is an $\alpha_{L}$-set of $T$, this implies that $U(T) \subseteq I^{c}$. By Theorem 1, we have that

$$
|I|= \begin{cases}\left\lfloor\frac{2(3 k+2)-1}{3}\right\rfloor=2 k+1, & \text { if } n=3 k+2, \\ \left\lfloor\frac{2(3 k+1)-1}{3}\right\rfloor=2 k, & \text { if } n=3 k+1, \\ \left.\mid \frac{2(3 k)-1}{3}\right\rfloor=2 k-1, & \text { if } n=3 k .\end{cases}
$$

Hence we obtain that $\left|I^{c}\right|=n-|I|=k+1$. Let $B=I-L(T)=\left\{z_{1}, z_{2}, \cdots, z_{b}\right\}$. Note that $N_{T}\left(z_{i}\right) \subseteq I^{c}$ and $\left|N_{T}\left(z_{i}\right)\right| \geq 2$ for every $i$. In addition, $\left|N_{T}\left(z_{i}\right) \cap N_{T}\left(z_{j}\right)\right| \leq 1$, these imply that $\left|I^{c}\right| \geq\left|\bigcup_{i=1}^{b} N_{T}\left(z_{i}\right)\right| \geq b+1$. Thus we obtain that $|B|=b \leq\left|I^{c}\right|-1=k$. It follows that

$$
\begin{aligned}
\left|I^{c} \backslash U(T)\right| & =\left|I^{c}\right|-|U(T)|=\left|I^{c}\right|-|L(T)| \\
& =\left|I^{c}\right|-(|I|-b) \leq n-2|I|+k \\
& = \begin{cases}(3 k+2)-2(2 k+1)+k=0, & \text { if } n=3 k+2, \\
(3 k+1)-2(2 k)+k=1, & \text { if } n=3 k+1, \\
(3 k)-2(2 k-1)+k=2, & \text { if } n=3 k .\end{cases}
\end{aligned}
$$

This completes the proof.

Lemma 6 Let $T \in \mathscr{T}(n)$ be a tree of order $n \equiv 2(\bmod 3)$ with an $\alpha_{L}$-set I. Suppose that $T^{\prime}$ is obtained from $T$ by Operation O1, then $T^{\prime} \in \mathscr{T}(n+1)$ is a tree of order $n+1$ and $I_{O 1}$ is an $\alpha_{L}$-set of $T^{\prime}$.

Proof. Suppose that $T \in \mathscr{T}(n)$ is a tree of order $n \equiv 2(\bmod 3)$ with an $\alpha_{L}$-set $I$, by Lemma 5 , then 
$I^{c}=U(T)$. Let $T^{\prime}$ be the tree obtained from $T$ by Operation O1. Since $u \in I$, this implies that $u$ is not a support vertex of $T$ and $T^{\prime}$ is a tree of order $n+1$ without duplicated leaves. On the other hand, $I_{O 1}$ is an independent set of $T^{\prime}$ with $L\left(T^{\prime}\right) \subseteq I_{O 1}$ such that $\left\lfloor\frac{2(n+1)-1}{3}\right\rfloor \geq \alpha\left(T^{\prime}\right) \geq\left|I_{O 1}\right|=|I|=\left\lfloor\frac{2 n-1}{3}\right\rfloor=\left\lfloor\frac{2(n+1)-1}{3}\right\rfloor$, where $n \equiv 2$ (mod 3). Hence $\alpha\left(T^{\prime}\right)=\left\lfloor\frac{2(n+1)-1}{3}\right\rfloor$. In conclusion, $T^{\prime} \in \mathscr{T}(n+1)$ is a tree of order $n+1$ with an $\alpha_{L}$-set $I_{O 1}$.

Lemma 7 Let $T \in \mathscr{T}(n)$ be a tree of order $n \equiv 0,1(\bmod 3)$ with an $\alpha_{L}$-set I such that $\left|I^{c}-U(T)\right| \geq 1$. If $T^{\prime}$ is obtained from $T$ by Operation O2, then $T^{\prime} \in \mathscr{T}(n+1)$ is a tree of order $n+1$ and $I_{O 2}$ is an $\alpha_{L}$-set of $T^{\prime}$.

Proof. Note that such a tree $T$ exists, as, for instance, the tree in Figure 1 is as desired. If $T \in \mathscr{T}(n)$ is a tree of order $n \equiv 0,1$ (mod 3) with an $\alpha_{L}$-set $I$ such that $\left|I^{c}-U(T)\right| \geq 1$. Let $T^{\prime}$ be the tree obtained from $T$ by Operation O2. Since $u$ is not a support vertex of $T$, this implies that $T^{\prime}$ is a tree of order $n+1$ without duplicated leaves. And $I_{O 2}$ is an independent set of $T^{\prime}$ with $L\left(T^{\prime}\right) \subseteq I_{O 2}$ such that $\left\lfloor\frac{2(n+1)-1}{3}\right\rfloor \geq \alpha\left(T^{\prime}\right) \geq\left|I_{O 2}\right|=|I|+1=\left\lfloor\frac{2 n-1}{3}\right\rfloor+1=\left\lfloor\frac{2(n+1)-1}{3}\right\rfloor$, where $n \equiv 0,1$ (mod 3). Hence $\alpha\left(T^{\prime}\right)=\left\lfloor\frac{2(n+1)-1}{3}\right\rfloor$. In conclusion, $T^{\prime} \in \mathscr{T}(n+1)$ is a tree of order $n+1$ with an $\alpha_{L}$-set $I_{O 2}$.

Lemma 8 Let $T \in \mathscr{T}(n)$ be a tree of order $n \equiv 1,2(\bmod 3)$ with an $\alpha_{L}$-set I. If $T^{\prime}$ is obtained from $T$ by Operation O3, then $T^{\prime} \in \mathscr{T}(n+2)$ is a tree of order $n+2$ and $I_{O 3}$ is an $\alpha_{L}$-set of $T^{\prime}$.

Proof. Note that $T^{\prime}$ is a tree of order $n+2$ without duplicated leaves. And $I_{O 3}$ is an independent set of $T^{\prime}$ with $L\left(T^{\prime}\right) \subseteq I_{\text {Оз }}$ such that $\left\lfloor\frac{2(n+2)-1}{3}\right\rfloor \geq \alpha\left(T^{\prime}\right) \geq\left|I_{O 3}\right|=|I|+1=\left\lfloor\frac{2 n-1}{3}\right\rfloor+1=\left\lfloor\frac{2(n+2)-1}{3}\right\rfloor$, where $n \equiv 1,2$ (mod 3). Hence $\alpha\left(T^{\prime}\right)=\left\lfloor\frac{2(n+2)-1}{3}\right\rfloor$. In conclusion, $T^{\prime} \in \mathscr{T}(n+2)$ is a tree of order $n+2$ with an $\alpha_{L^{-}}$ set $I_{O 3}$.

Lemma 9 Let $T \in \mathscr{T}(n)$ be a tree of order $n \geq 4$ with an $\alpha_{L}$-set I. If $T^{\prime}$ is obtained from $T$ by Operation $\mathrm{O} 4$, then $T^{\prime} \in \mathscr{T}(n+3)$ is a tree of order $n+3$ and $I_{O 4}$ is an $\alpha_{L}$-set of $T^{\prime}$.

Proof. Note that $T^{\prime}$ is a tree of order $n+3$ without duplicated leaves. And $I_{O 4}$ is an independent set of $T^{\prime}$ with $L\left(T^{\prime}\right) \subseteq I_{O 4}$ such that $\left\lfloor\frac{2(n+3)-1}{3}\right\rfloor \geq \alpha\left(T^{\prime}\right) \geq\left|I_{O 4}\right|=|I|+2=\left\lfloor\frac{2 n-1}{3}\right\rfloor+2=\left\lfloor\frac{2(n+3)-1}{3}\right\rfloor$. Hence $\alpha\left(T^{\prime}\right)=\left\lfloor\frac{2(n+3)-1}{3}\right\rfloor$. In conclusion, $T^{\prime} \in \mathscr{T}(n+3)$ is a tree of order $n+3$ with an $\alpha_{L}$-set $I_{O 4}$.

Let $C$ be the class of all trees obtained from $P_{4}$ or $P_{5}$ by a finite sequence of Operations O1-O4. Suppose that $\mathscr{T}=\bigcup_{n \geq 4} \mathscr{T}(n)$, we will show that $\mathscr{T}=\mathscr{C}$.

Theorem $2 T$ is in $\mathscr{C}$ if and only if $T$ is in $\mathscr{T}$.
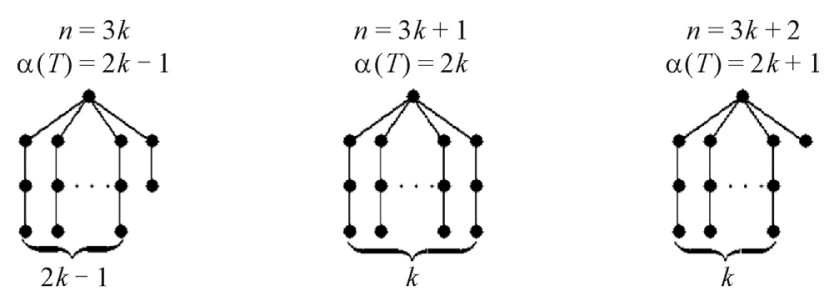

Figure 1. The trees $T$ with $\alpha(T)=\left\lfloor\frac{2 n-1}{3}\right\rfloor$. 
Proof. If $T$ is in $\mathscr{C}$, by Lemmas 6, 7, 8 and 9, then $T$ is in $\mathscr{T}$. Now, we want to show the converse by contradiction. Suppose to the contrary that there exists a tree $T \in \mathscr{T}$ and $T \notin \mathscr{C}$ such that $|T|$ is as small as possible. We can see that $|T| \geq 7$. Let $P: x-y-z-\cdots$ be a longest path of $T$. Then $N_{T}(y)=\{x, z\}$ and $T^{\prime}=T-N_{T}[x]$ is a tree of order $n^{\prime}=n-2$. We consider two cases.

Case 1. $T^{\prime}$ has no duplicated leaves.

For an $\alpha_{L}$-set $I$ of $T, I^{\prime}=I-\{x\}$ is an independent set of $T^{\prime}$, this implies that $\alpha\left(T^{\prime}\right) \geq\left|I^{\prime}\right|=\alpha(T)-1$. By Theorem 1, we have that $\left\lfloor\frac{2 n-1}{3}\right\rfloor-1=\left\lfloor\frac{2 n-4}{3}\right\rfloor=\alpha(T)-1 \leq \alpha\left(T^{\prime}\right) \leq\left\lfloor\frac{2(n-2)-1}{3}\right\rfloor=\left\lfloor\frac{2 n-5}{3}\right\rfloor \leq\left\lfloor\frac{2 n-4}{3}\right\rfloor$. Then $\alpha\left(T^{\prime}\right)=\left\lfloor\frac{2(n-2)-1}{3}\right\rfloor=\left\lfloor\frac{2 n-1}{3}\right\rfloor-1$ and $n \equiv 0,1(\bmod 3)$. This follows that $T^{\prime} \in \mathscr{T}\left(n^{\prime}\right)$, where $n^{\prime}=n-2 \equiv 1,2 \quad(\bmod 3)$, by hypothesis, $T^{\prime} \in \mathcal{C}$. Note that $T$ can be obtained from $T^{\prime}$ by Operation O3, this implies that $T \in \mathscr{C}$, which is a contradiction.

Case 2. $T^{\prime}$ has duplicated leaves $z$ and $z^{\prime}$.

Let $T^{\prime \prime}=T^{\prime}-\{z\}$. Then $T^{\prime \prime}$ is a tree of order $n-3$. Since $z^{\prime}$ is a leaf of $T$, this implies that $z$ and $z^{\prime}$ are in every $\alpha_{L}$-set of $T$. For an $\alpha_{L}$-set $I$ of $T, I^{\prime \prime}=I-\{x, z\}$ is an independent set of $T^{\prime \prime}$, thus $\alpha\left(T^{\prime \prime}\right) \geq\left|I^{\prime \prime}\right|=\alpha(T)-2$. By Theorem 1 , we have that $\left\lfloor\frac{2 n-1}{3}\right\rfloor-2=\left\lfloor\frac{2 n-7}{3}\right\rfloor=\alpha(T)-2 \leq \alpha\left(T^{\prime \prime}\right) \leq\left\lfloor\frac{2(n-3)-1}{3}\right\rfloor=\left\lfloor\frac{2 n-7}{3}\right\rfloor$. Then $\alpha\left(T^{\prime \prime}\right)=\left\lfloor\frac{2(n-3)-1}{3}\right\rfloor$. This follows that $T^{\prime \prime} \in \mathscr{T}\left(n^{\prime \prime}\right)$, where $n^{\prime \prime}=n-3$, by hypothesis, $T^{\prime \prime} \in \mathcal{C}$. Note that $T$ can be obtained from $T^{\prime \prime}$ by Operation O4, this implies that $T \in \mathscr{C}$, which is a contradiction.

By Cases 1 and 2, we conclude that $T$ is in $\mathscr{T}$, then $T$ is in $\mathscr{C}$.

Now, we obtain the main theorem in this paper.

Theorem 3 Suppose that $T$ is a tree of order $n \geq 4$ without duplicated leaves, then $\alpha(T) \leq\left\lfloor\frac{2 n-1}{3}\right\rfloor$. Furthermore, the equality holds if and only if $T \in \mathscr{C}$.

\section{References}

[1] Bondy, J.A. and Murty, U.S.R. (1976) Graph Theory with Application. North-Holland, New York.

[2] Harant, J. (1998) A Lower Bound on the Independence Number of a Graph. Discrete Mathematics, 188, $239-243$. http://dx.doi.org/10.1016/S0012-365X(98)00048-X

[3] Hattingh, J.H., Jonack, E., Joubert, E.J. and Plummer, A.R. (2007) Total Restrained Domination in Trees. Discrete Mathematics, 307, 1643-1650. http://dx.doi.org/10.1016/j.disc.2006.09.014

[4] Jou, M.-J. (2010) Dominating Sets and Independent Sets in a Tree. Ars Combinatoria, 96, 499-504.

[5] Jou, M.-J. (2010) Upper Domination Number and Domination Number in a Tree. Ars Combinatoria, 94, 245-250.

[6] Luo, R. and Zhao, Y. (2006) A Note on Vizing’s Independence Number Conjecture of Edge Chromatic Critical Graphs, Discrete Mathematics, 306, 1788-1790. http://dx.doi.org/10.1016/j.disc.2006.03.040 